\title{
Reflexiones sobre Filosofía y la Realidad Científica
}

\author{
Reflections on Philosophy and Scientific Reality
}

\author{
Dr. Luis Alberto Hernández-Bowen ${ }^{1}$
}

\section{2 \\ Edición Especial: ENSAYOS}

Recibido: 11 de julio de 2020

Aceptado: 20 de agosto de 2020

Publicado: 23 de agosto de 2020

Institución:

而 ${ }^{1}$ Universidad Yacambú

Venezuela

E-mail / ORCID:

vcrinvepuny@uny.edu.ve

https://orcid.org/0000-0001-8597-9411

Citar así:

CGAPA / IEEE

Citar así: Hernández-Bowen, L. (2020). Reflexiones sobre Filosofía y la Realidad Científica. Revista Tecnológica-Educativa Docentes 2.0, 8(2), 34-38, DOI: https://doi.org/10.37843/rted.v8i2.151

L. Hernández-Bowen. "Reflexiones sobre Filosofía y la Realidad Científica”. RTED, 8, No. 2, pp. 34-38, ago. 2020

\section{Resumen}

El presente ensayo tuvo como objetivo generar algunas reflexiones ilustradoras de esquemas filosóficos de mérito respecto al ámbito científico, siendo en los entornos académicos universitarios doctorales donde se perciben corrientes que se han convertido en tendencia, tales como el enfoque postestructuralista, el relativismo, investigaciones fenomenológicas hermenéuticas, entre otras. Hoy en día, cobra especial trascendencia la importancia de la filosofía respecto al existencialismo humanista en contextos científicos, abarcando espacios importantes para producir saberes en las orientaciones positivistas como postpositivistas donde se enmarca la hermenéutica filosófica de Gadamer. Todo ello, evidencia una corriente anticientifista basada en el manifiesto de misma índole impulsado por Wilhelm Dilthey. En buena medida esto responde a una necesaria reflexión sobre algunas tendencias filosóficas que deben fungir como criterio de validez científica, pese a existir el enfoque basado en una epistemología naturalizada desde donde se concibe desvincular ciencia y filosofía. En este sentido, parece pertinente retomar la necesaria labor de reflexionar sobre estas tendencias filosóficas fundamentales para lograr innovaciones dentro de los planos del conocimiento desde los entornos universitarios doctorales, pero con estándares aceptables vinculados a una alta contratación teórica como tarea ineludible. Hasta no entender una necesaria relación de complementariedad entre investigación respecto a las corrientes filosóficas, seguiremos dando cabida a procesos de carentes de estándares aceptables.

Palabras claves: Filosofía, ciencia, realidad.

\section{Abstract}

The objective of this essay was to generate some illustrative reflections of philosophical schemes of merit regarding the scientific field, being in doctoral university academic environments where currents that have become a trend are perceived, such as the poststructuralist approach, relativism, phenomenological research hermeneutics, among others. Today, the importance of philosophy with respect to humanistic existentialism in scientific contexts takes on special significance, encompassing important spaces to produce knowledge in positivist and postpositivist orientations where Gadamer's philosophical hermeneutics is framed. All of this shows an anti-scientific trend based on the manifesto of the same nature promoted by Wilhelm Dilthey. To a large extent, this responds to a necessary reflection on some philosophical trends that should serve as a criterion of scientific validity, despite the existence of an approach based on a naturalized epistemology from which it is conceived to unlink science and philosophy. In this sense, it seems pertinent to retake the necessary work of reflecting on these fundamental philosophical tendencies to achieve innovations within the planes of knowledge from doctoral university environments, but with acceptable standards linked to a high theoretical hiring as an unavoidable task. Until we understand a necessary relationship of complementarity between research with respect to philosophical currents, we will continue to accommodate processes lacking acceptable standards.

Keywords: Philosophy, science, reality. 


\section{Reflexiones sobre Filosofía y la Realidad Científica}

\section{Introducción}

Hoy en día, cobra especial trascendencia la importancia de la filosofía respecto al existencialismo humanista en contextos científicos, abarcando espacios importantes para producir saberes en las orientaciones positivistas como postpositivistas o postestructuralistas donde se enmarca la hermenéutica filosófica de Gadamer.

El origen del pensamiento filosófico se ubica en la era arcaica de Grecia, donde se buscaba dar explicación a los fenómenos naturales movidos por una búsqueda de lo que es verdadero, pero al inicio existencial del hombre era abordado desde el mito, por cuanto mito y logos. Estos espacios fueron origen del objeto mismo de lo antológico, como inicio por estudiar el ser o como diría Heidegger el ser en el mundo, pero posteriormente fue centrándose en el posicionamiento del sujeto respecto a realidades sobre las cuales dentro de los parámetros de un estudio doctoral se referiría al objeto de estudio.

Es lamentable ver como influencia del postpositivismo, las orientaciones cualitativas, autores como Edgar Morín han trastocado el entendimiento de los abordajes ontológicos llevando a considerarlos una comunicación con el sujeto, lo cual se evidencia en los entornos universitarios. Bien sabemos, una teoría filosófica al menos debe considerar aspectos como los principios sobre los cuales esta subyace, los principios fundamentales de lógico aristotélicos, entre otros para sustentar sus ideas.

En este sentido, se observa dentro de los entornos universitarios posicionamientos en lo antológico sin considerar las bases de las proposiciones fundamentales o fundacionalismo como son los linderos realistas e idealistas, dentro de los planos del conocimiento estas corrientes constituyen una necesaria referencia en lo antológico. Incluso, podría afirmarse el desconocimiento a las corrientes existentes dentro de cada uno de estas.

Se desconoce los parámetros más necesarios para contrastar ideas como el cientifismo, como tesis fundamental es encarar los problemas del conocimiento adoptando el método científico, o llamado el espíritu o actitud científica. El cientifismo constituye una actitud normal de todo investigador.

Reflexionar sobre este aspecto permite pensar sobre constelaciones de autores cuya motivación ha sido el desarrollo de estas ideas, los cuales se pueden desagregar en tres categorías: Los cientifistas, los anticientifistas, los semicientifistas. Los cientifistas como el polibata Nicolas de Condorcet de fines del siglo XVIII, el psicólogo, así como sociólogo Piaget, el sociólogo Robert Merton, mientras que Hegel, Nietzsche, Heidegger pertenecen al grupo anticientifista, en cambio Kant, Comte, Marx elogiaron las orientaciones racionales como universalidad científica, pero permanecieron encadenados a dogmas anticientíficos el fenomenismo de Kant, Comte, el hermetismo dialectico en el caso de Marx.

Esto se debe como refiere el Dr. Bunge la concepción de ciencia y cientifismo como dos bestias del partido oscurantistas incomprendidas en los escenarios universitarios. A este tenor se evidencia dentro de las exposiciones; así en abordajes filosóficos como indica el Dr. José Padrón Guillen no se cuida o responde a una familia de autores, citando a Heidegger y Husserl concomitantemente, pese a poseer concepciones fenomenológicas contradictorias, al surgir una teoría en aversión a la otra. Esto redunda en un reduccionismo ontológico que en años recientes ha caracterizado el quehacer sociológico, psicología evolutiva, neuroeconomía, así como demás neuros de moda por demás meramente programáticos.

$\mathrm{Ni}$ siquiera comprenden muchas veces estas corrientes asumidas, ignorando hechos destacados del filósofo Alemán Dilthey quien en 1883 formuló su manifiesto anticientifista, con un componente ontológico desde el cual todos los hechos sociales son hechos espirituales por sobre lo material, para este autor los hechos sociales son ciencias del espíritu, por ende, ameritan un método propio basado en comprender y explicar. Comprender intuitiva o 


\section{Reflexiones sobre Filosofía y la Realidad Científica}

empáticamente el sentir o pensamiento del actor, por cuanto concibe las ciencias sociales como estudio de individuos no de grupos.

Lo mismo ocurre con las orientaciones epistemológicas, por cuanto en Grecia se basaba en los postulados del racionalismo, es decir consideró el conocimiento verdadero al derivado de procesos mentales del razonamiento humano, negando así tal validez a los conocimientos empíricos. Por ello no es de extrañar al evolucionar, el pensamiento filosófico griego fuera hacia corrientes basadas en el pensamiento, como forma de explicación de lo real articulado con el estudio del conocimiento.

Fundamentalmente, hablar de filosofía e investigación implica directamente referir al ámbito científico, por cuanto los procesos de investigación deben atender a estándares válidos para garantizar en sus aportes, parámetros comprobados desde donde se oriente su accionar como generación de conocimientos válidos, aspecto directamente relacionado con la epistemología. Es claro hoy en día dentro de los entornos doctorales desarrollados en los países no anglosajones, es necesario buscar conocimientos epistémicos, marcando distancia del conocimiento vulgar o gnoseológico. Ese conocimiento es parte de los abordajes de las teorías filosóficas.

Ahora bien, no podemos afirmar en forma absoluta como generalizada en las tendencias actuales al referir a una epistemología es hablar de filosofía, toda vez existe una tendencia sobre desvincular epistemología de filosofía. En un primer momento, conocemos el vínculo entre epistemología, filosofía vinculada al concepto de filosofía de la ciencia, pero luego esta relación ha cambiado, siendo ratificado en tendencias recientes por el surgimiento de la epistemología naturalizada, liderada por Quine enmarcada en el posicionamiento desde una epistemología naturalizada, cognitiva, evolucionista.

Es por ello necesario realizar el abordaje de esta temática, a fin de identificar las orientaciones conceptuales para sustentan las reflexiones realizadas sobre el particular.
En este sentido, al inicio del artículo, fue abordado el aspecto principal para esta labor fundamental que cumple toda investigación como debería ser, brindar soluciones a los problemas existentes dentro del contexto social.

Pero existen otros aspectos integrados al propósito de investigación, como el descubrimiento de algo desconocido Sierra (1991), solución de un problema de conocimiento (Sabino, 2002, p. 34). Sobre este particular concluye Arias (2012) que la investigación implica: “a) Descubrimiento de algún aspecto de la realidad. b) La producción de un nuevo conocimiento, el cual puede estar dirigido a incrementar los postulados teóricos de una determinada ciencia (investigación pura o básica; o puede tener una aplicación inmediata en la solución de problemas piráticos (investigación aplicada)". (p. 12).

Siendo estos los propósitos implícitos en la función de investigar, vemos como el papel de la filosofía ha venido deslastrándose de los conceptos de ciencia, epistemología y por ende de investigar. Al respecto, afirma Padrón (2013) al referirse a las nociones de filosofía como epistemología:

\begin{abstract}
$\mathrm{Su}$ presupuesto conceptual es que se trata de una rama incluida en la Filosofía, sinónimo, hasta donde puede inferirse, de Filosofía de la Ciencia. El hecho de estar incluida dentro de la Filosofía tiene muchas implicaciones, una de las cuales resulta clave para poder entender la epistemología evolucionista. Como sabemos, la mayoría de las disciplinas que actualmente consideramos como Ciencias autónomas en un principio pertenecieron a la filosofía, pero abandonaron esa cuna desde el momento en que cada una de ellas encontró su universo de correlatos empíricos y sus esquemas metodológicos de recolección de datos, de construcción teórica y de contrastación (fuera formal o empírica). (p. 23).
\end{abstract}

Refiere al ejemplo de ciencias como la física, siendo en un principio vinculada a corrientes filosóficas, pero luego encontró su autonomía dentro de los estándares científicos, adoptando herramientas como mecanismo para 


\section{Reflexiones sobre Filosofía y la Realidad Científica}

dar solución a su propio desarrollo. Sobre este particular Padrón (2013) indica:

\begin{abstract}
Así, por ejemplo, la Física actual en un principio, hace muchos siglos, era "Ontología", una rama adscrita a la Filosofía. Pero una vez que tal disciplina logró delimitar el universo de hechos que pretendía explicar y una vez que definió sus propias operaciones experimentales y de laboratorio como medios de búsqueda y de prueba, dejó de pertenecer a la Filosofía y se estableció como Ciencia. (p. 24).
\end{abstract}

En el mismo sentido refiere al caso de la psicología, por cuanto alcanza su autonomía desde una vertiente filosófica cuando desarrolla sus propias herramientas y logra su desarrollo en base a historia científica, así como de sus propósitos comunes. Al respecto Padrón (2013) relaciona sobre el particular:

\begin{abstract}
La última que abandonó la cuna de la Filosofía fue la Psicología, cuando, según se reconoce en todos los documentos, Wundt fundó el primer laboratorio de Psicología Experimental en Leipzig, en 1878. Lo esencial de poder montar un laboratorio y de realizar unos experimentos es que ya quedaba delimitado el universo de hechos $o$ el correlato empírico correspondiente a la Psicología. Desde entonces, pasó a ser considerada una Ciencia autónoma y los centros de estudios empíricos y experimentales en psicología se fueron extendiendo por todo el mundo. (p. 24).
\end{abstract}

Ahora bien, ciertamente hasta los comienzos de la antigüedad clásica, tanto los términos filosofía como ciencia estuvieron fusionadas, lo científico era una rama filosófica según Aristóteles. Luego alrededor del siglo XVI se independizan, cobran autonomía las ciencias naturales y, pero en el siglo 19 las ciencias humanas.

El problema actual de esta vinculación anteriormente fusión entre filosofía en cuento a ciencia, ahora ha devenido en una relación conflictiva entre científicos y filósofos, si consideramos una rama de filósofos como anticientíficos dentro del romanticismo del siglo XIX como del siglo XX con Nietzsche y
Heidegger con la propuesta del postestructuralismo o postpositivismo. Muestra de esta actitud anticientifista es el manifiesto generado por Dilthey este manifiesto tenía un componente ontológico, adicional a uno metodológico.

En el ontológico considera los hechos sociales como hechos espirituales negando los materiales. Posee un componente socio metodológico en cuento a tesis ontológica es obvio los estudios sociales son ciencias del espíritu, no ciencias de lo material por ende exigen un método propio, este método seria la comprensión e interpretación, más no una explicación en el sentido científico.

Por el otro lado, tenemos ciencias contrarias a tendencias filosóficas en el siglo XIX como el positivismo, así en el siglo XX el neopositivismo del Círculo de Viena. Pero, por el contario la relación entre investigación científica con pensamiento filosófico es fundamental, por cuanto es necesario complementariedad entre sí. Debemos asumir una filosofía para el análisis de los resultados que aporte el investigador científico, así como el científico no puede obviar los procesos filosóficos, por cuanto le brinda un contexto una cosmovisión.

Una vez se retome relaciones armoniosas como complementarias entre investigadores respecto a filósofos, lograran entender las corrientes filosóficas como validadoras de la ciencia e investigación. En este sentido, cuando siendo parte esencial de investigar generar procesos de indagación, los conocimientos generados deben poseer un basamento epistemológico al cual considero nuevamente el quehacer filosófico le brinda un soporte de validación porque lo ha reflexionado. En este sentido, estimo debemos desvincular los procesos de investigación de una metodología de la investigación para desarrollarlos desde las bases que brindan, tanto epistemología como filosofía, sobre la base de una necesaria validez del conocimiento generado como al proceso de investigación desarrollado.

Sobre este base, Padrón (2007) ha propuesto los enfoques epistemológicos en los 


\section{Reflexiones sobre Filosofía y la}

Realidad Científica

cuales se puede enmarcar cualquier método y proceso de investigación, combinando las corrientes ontológicas como epistemológicas en función de brindar los cuatro enfoques como lo son en empírico-inductivista, racionalistadeductivista, el vivencial experiencialista como socio simbolista, aspectos que claramente se encuentran alineados con la filosofía como contexto en función de fundamentar el desarrollo de las investigaciones.

\section{Conclusión}

Como sabemos, la ciencia y la investigación se encargan de dar respuesta al abordaje sobre ¿Cuánto es el tiempo para un rayo llegar del sol al planeta tierra, pero una filosofía actual se ocupa en ese caso de abordar como definir el tiempo en todo lugar como en todo momento, aspecto constitutivo del objeto de la filosofía? Los procesos de investigación abordan problemas sobre la distancia dentro de la física, como medirla, entre otros, en cambio la filosofía complementa esta desde su autonomía de objeto, cuando explica la concepción de distancia.

Vemos aquí expresado el ejemplo de vinculación complementaria entre investigación vs filosofía. La investigación en cuanto a ciencia no puede dejar de plantearse problemas filosóficos, es el caso de investigaciones psicológicas donde se desarrollan estudios sobre la mente humana por ejemplo desde una psicología cognitiva que atienda problemas de aprendizaje vinculados a los estilos de pensamiento, pero el pensamiento del filósofo se encarga de reflexionar sobre qué debemos asumir como mente.

Finalmente, hasta no entender una necesaria relación de complementariedad entre investigación respecto a las corrientes filosóficas, seguiremos dando cabida a procesos de investigación carentes de estándares aceptables, por ende, diluyendo el esfuerzo impreso en ellos, contrarios estos al espíritu para solventar el desarrollo de sociedades enteras como función de los procesos de investigación.

\section{Referencias}

Arias, F (2012). El Proyecto de Investigación.6ta Edición. Editorial Episteme. Caracas. República Bolivariana de Venezuela.

Cervo, A \& Bervian, P. (1989). Metodología Cientifica. Bogotá: McGraw-Hill.

Sierra-Bravo, R (1991). Técnicas de investigación Social. ( $7^{\circ}$ ed). Paraninfa.

Sabino, C (2002). El Proceso de investigación. Panapa.

Padrón J. (2007). Tendencias epistemológicas de la investigación cientifica en el Siglo XXI. Entre Temas. Recuperado de: https://www.moebio.uchile.cl/28/padron.html

Padrón, J. (2013). Epistemología Evolucionista. Una visión integral. Recuperado de: https://www.grin.com/document/412021. 